\title{
Active Magnetoelectric Motion Sensing: Examining Performance Metrics with an Experimental Setup
}

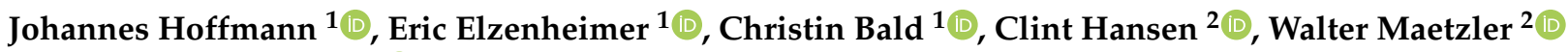 \\ and Gerhard Schmidt ${ }^{1, *(D)}$ \\ 1 Institute of Electrical Engineering and Information Technology, Faculty of Engineering, Kiel University, \\ 24143 Kiel, Germany; jph@tf.uni-kiel.de (J.H.); ee@tf.uni-kiel.de (E.E.); cbal@tf.uni-kiel.de (C.B.) \\ 2 Department of Neurology, Kiel University, 24105 Kiel, Germany; c.hansen@neurologie.uni-kiel.de (C.H.); \\ w.maetzler@neurologie.uni-kiel.de (W.M.) \\ * Correspondence: gus@tf.uni-kiel.de; Tel.: +49-431-880-6125
}

check for updates

Citation: Hoffmann, J.; Elzenheimer, E.; Bald, C.; Hansen, C.; Maetzler, W.; Schmidt, G. Active Magnetoelectric Motion Sensing: Examining Performance Metrics with an Experimental Setup. Sensors 2021, 21, 8000. https://doi.org/10.3390/ s21238000

Academic Editor: Angelo Maria Sabatini

Received: 26 October 2021

Accepted: 28 November 2021

Published: 30 November 2021

Publisher's Note: MDPI stays neutral with regard to jurisdictional claims in published maps and institutional affiliations.

Copyright: (c) 2021 by the authors. Licensee MDPI, Basel, Switzerland. This article is an open access article distributed under the terms and conditions of the Creative Commons Attribution (CC BY) license (https:/ / creativecommons.org/licenses/by/ $4.0 /)$.

\begin{abstract}
Magnetoelectric (ME) sensors with a form factor of a few millimeters offer a comparatively low magnetic noise density of a few $\mathrm{pT} / \sqrt{\mathrm{Hz}}$ in a narrow frequency band near the first bending mode. While a high resonance frequency ( $\mathrm{kHz}$ range) and limited bandwidth present a challenge to biomagnetic measurements, they can potentially be exploited in indirect sensing of non-magnetic quantities, where artificial magnetic sources are applicable. In this paper, we present the novel concept of an active magnetic motion sensing system optimized for ME sensors. Based on the signal chain, we investigated and quantified key drivers of the signal-to-noise ratio (SNR), which is closely related to sensor noise and bandwidth. These considerations were demonstrated by corresponding measurements in a simplified one-dimensional motion setup. Accordingly, we introduced a customized filter structure that enables a flexible bandwidth selection as well as a frequency-based separation of multiple artificial sources. Both design goals target the prospective application of ME sensors in medical movement analysis, where a multitude of distributed sensors and sources might be applied.
\end{abstract}

Keywords: motion tracking; magnetoelectric sensors; artificial fields

\section{Introduction}

Magnetic sensing is well established in movement analysis [1,2] as most inertial measurement units (IMUs) contain 3D magnetometers to determine the unit's orientation in the horizontal plane (compass). Common scientific applications include human movement analysis, where medical doctors use motion tracking systems in both diagnosis and therapy of neurodegenerative disorders with movement-related symptoms: sensors are commonly applied in a lab-based assessment to examine early motor markers of Parkinson's disease [3]. Patient monitoring in the home setting is another emerging approach that relies heavily on wearable sensor technology [4,5].

Active magnetic motion tracking shows the potential to be a supplemental source of reference data [6]. In comparison to the passive magnetic method, it utilizes artificial magnetic fields of excitation coils in combination with tracking algorithms to obtain the relative position and orientation data between source and sensor [7]. Integrated commercial magnetometers based on Hall-effect sensors are well-suited for pure geomagnetic applications (up to $50 \mu \mathrm{T}$ ), as they offer sufficient noise performance (e.g., below $0.3 \mu \mathrm{T}$ for BMX005, Bosch Sensortec [8]) at low cost and small integration size. For active magnetic motion tracking, sensor performance is of much greater concern, as actuators (excitation coils) consume significant amounts of power to generate magnetic fields, which is noticeable in the large required currents (e.g., $1.5 \mathrm{~A}$ [6]). A low power consumption is generally desirable as it improves runtime for battery-powered applications. In essence, the required power to reach a given signal-to-noise ratio (SNR) at a given distance (and orientation) can be 
significantly decreased by the selection of a performant sensor type in combination with optimized transmission and readout schemes.

In this contribution, we employ magnetoelectric (ME) delta-E-effect sensors [9] in direct detection of the first bending mode (approximately $7.7 \mathrm{kHz}$ ) with a magnetic noise density below $10 \mathrm{pT} / \sqrt{\mathrm{Hz}}$ in resonance. Assuming mono-frequent signals at a sensor-optimized frequency, the sensors perform in the same order of magnitude as fluxgate sensors (e.g., FL1-100, Stefan Mayer Instruments: $20 \mathrm{pT} / \sqrt{\mathrm{Hz}}$ at $1 \mathrm{~Hz}$ [10]) and slightly inferior to total field optically pumped magnetometers (e.g., QTFM, QuSpin: below $1 \mathrm{pT} / \sqrt{\mathrm{Hz}}$ [11]). However, OPMs require heating power (e.g., total field OPM, Twinleaf: $0.7 \mathrm{~W}$ [12]) for laser and cell temperature stabilization, which might be undesirable in body-worn setups. Better performing sensors like superconducting quantum interference devices (SQUIDs) are not considered here, as they bring the significant drawbacks of supercooling (SQUIDs) or magnetically shielded operation (zero field OPMs). Aside from sensor noise, requirements differ heavily between applications as the term "motion sensing" is used interchangeably in indoor tracking/navigation, localization, and motion tracking of humans. Relevant performance metrics include measurement volume, (stationary) spatial accuracy, and update rate/bandwidth.

Indoor tracking or navigation systems as in $[13,14]$ offer a measurement volume of multiple cubic meters aided by IMUs. In contrast, specialized devices for the tracking of medical instruments (e.g., Polhemus Viper [15]) target a much smaller volume and achieve sub-millimeter accuracy for the stationary case. Depending on actuator and sensor, different frequency ranges can be targeted: direct current (dc) systems have to compensate for the geomagnetic field and other stray fields (hard iron) [16]. Alternating current (AC) systems [15] might operate in the very low frequency (VLF) band from $3 \mathrm{kHz}$ to $30 \mathrm{kHz}$, which is also suitable for the delta-E sensor type. Such systems avoid the geomagnetic field and some DC stray fields but have to deal with eddy currents in nearby conductive materials [17]. Due to their propagation velocity at the speed of light and their wavelengths in the $10 \mathrm{~km}$ range, these VLF approaches do not suffer from multi-path propagation and the Doppler effect [7], which results in a straight forward channel model for the stationary case. However, this does not eliminate the impact of relative movement in the context of complex field geometries.

Motion tracking systems commonly (e.g., [13]) use stationary sources in combination with distributed sensors. As most systems use multiple (orthogonal) sources, some form of multiple-access technique has to be employed to divide the source signals at the sensor. The sources might therefore operate sequentially as in [6] (time division multiple access, TDMA), by applying spreading codes (code division multiple access, CDMA) [13] or in separate frequency bands (frequency division multiple access, FDMA), such as the magnetoelectric approach presented here. The operation of multiple interoperable transmitters and receivers potentially enables powerful tracking approaches, as each additional unit results in a multitude of new data points. For a number of $L$ sources and $M$ sensors, this results in $L \times M$ data points.

This article focuses on the sensing of magnetic fields where characteristics of excitation, field, sensor, and basic signal enhancement are considered. This does not include tracking and sensor fusion algorithms [14], which are typically the following processing stages and can contribute to a significantly improved overall performance.

This paper is structured as follows: Section 2 details the characterization of the components and their mathematical description in the system. Section 3 introduces the experimental setup. It also features the acquired signals for the single channel and the FDMA approach as well as noise measurements and derived performance metrics. Section 4 contains a conclusion and an outlook. 


\section{System Characterization and Simulation}

The magnetoelectric motion sensing system (Figure 1) consists of three subsystems:

(1) The transmitter generates a magnetic AC field by feeding a periodic excitation signal $g(n)$ (zero-mean, normalized power) through an amplifier to a coil.

(2) The receiver (1D sensor, fixed orientation) moves on a trajectory $\vec{r}(t)$ relative to the transmitter unit. Thus, it senses the excitation signal $g(t)$ weighted with the magnetic flux density $d(t)$ (desired signal) along the trajectory. This behavior resembles an amplitude modulation.

(3) The signal processing system's task is to estimate an unbiased $\hat{d}(k)$ in a frame-based fashion with the subsampled time index $k$. Therefore, prior knowledge on the sensor system (signal enhancement) and the excitation sequence (matched filter) is applied.

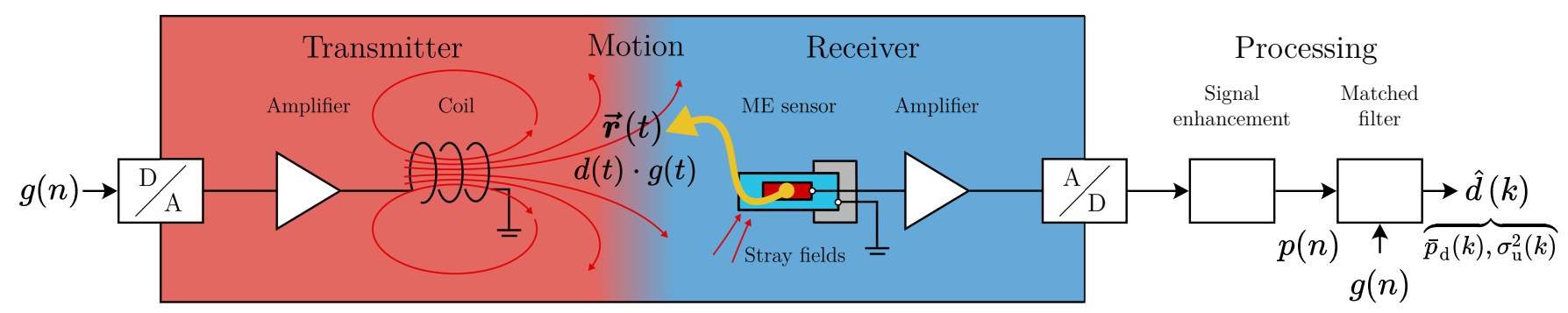

Figure 1. Magnetic motion sensing system overview.

The mathematical description of our approach is based on a distinction between the periodic excitation signal $g(n)$ and the discrete desired signal $d(n)$, which corresponds to a DC magnetic field with time variance induced by motion. The central component of the enhanced signal $p(n)$ is the modulation term (with minor deviations between discrete and continuous signals omitted). Undesired signal components like stray fields and sensor intrinsic noise are represented in the aggregated noise term $u_{\mathrm{p}}(n)$ :

$$
p(n)=d(n) g(n)+u_{\mathrm{p}}(n) .
$$

The matched filter separates the desired signal $d(n)$ from the excitation signal $g(n)$. Therefore, the output estimation is comprised of only the desired signal power $\bar{p}_{\mathrm{d}}(k)$ and the noise power $\sigma_{\mathrm{u}}^{2}(k)$ (zero-mean). Thus, the resulting signal-to-noise ratio (SNR) is independent of the excitation signal waveform:

$$
\operatorname{SNR}(k)=\frac{\bar{p}_{\mathrm{d}}(k)}{\sigma_{\mathrm{u}}^{2}(k)} .
$$

We introduce a position-dependent SNR for spatial performance considerations that only depends on the sensor position $\vec{r}$ at a given moment in time, without prior knowledge of the trajectory. Its definition requires multiple assumptions:

(1) The period of the highest frequency component in the desired signal $T_{\mathrm{d}}^{\max }$ is much longer than the temporal length of the matched filter $T_{\mathrm{mf}}$. This leads to a desired signal power $d^{2}(\vec{r})$ which is approximately constant within a frame.

(2) The noise power is both space- and time-invariant within the measurement area and duration.

$$
\operatorname{SNR}(\overrightarrow{\boldsymbol{r}})=\frac{d^{2}(\overrightarrow{\boldsymbol{r}})}{\sigma_{\mathrm{u}}^{2}}
$$

The following subsections focus on the contributions of the source and the sensor system regarding this metric in the system's operating frequency range near the sensor's resonance frequency $f_{0}$ of approximately $7.7 \mathrm{kHz}$. 


\subsection{Source Selection and Limitations}

As a setup with multiple homogeneous or linear gradient fields is difficult to employ in a scenario with distributed moving sources, we used small cylindrical air coils. Manufacturing was done by spooling approximately 80 windings $\left(N_{\mathrm{W}}\right)$ of $0.5 \mathrm{~mm}$ enameled copper wire on a 3D-printed plastic body. The medium radius $R$ was approximately $6.7 \mathrm{~mm}$.

According to Ampere's circuital law [17], the magnetic flux density of a conductor loop is driven by the current $I_{\mathrm{rms}}$. For a specified input voltage (voltage source) $I_{\mathrm{rms}}$ is limited by the electrical impedance of the coil, whose equivalent circuit is assumed here as a copper resistance in series with an inductance. Both parameters of the primarily used coil in the relevant frequency range were measured with a vector network analyzer (Bode 100, Omnicron Labs [18]). The resistance (Figure 2a) is close to $0.24 \Omega$ at $f_{0}$, while the inductance (Figure $2 \mathrm{~b}$ ) is almost constant with $24 \mu \mathrm{H}$. These comparatively low values have to be considered with regard to accurate current measurements.

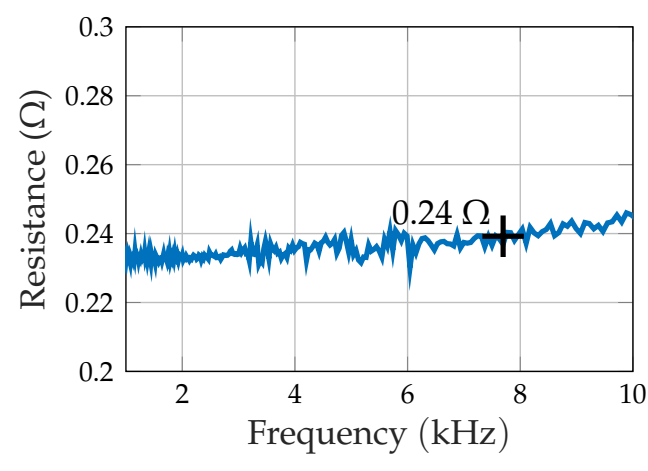

(a) Copper resistance.

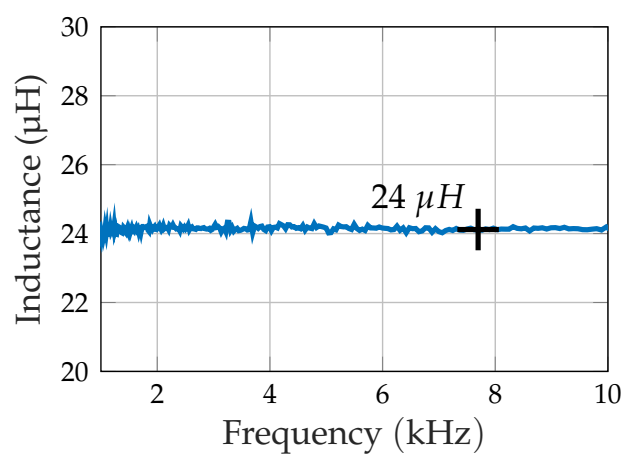

(b) Inductance.

Figure 2. Impedance of the primary excitation coil.

The resulting magnetic flux density $\vec{b}$ of the coil is also affected by its physical properties. As a universal approach, the law of Biot-Savart [17] can be applied for the field computation of an arbitrary ensemble of line conductors. While there are analytic solutions for a simple cylindrical helix coil [19], a more complex coil might require a computationally intensive discrete model.

The magnetic dipole equation [20] offers a lightweight alternative, assuming the displacement $r$ is much greater than the medium radius of the coil $R$. The actual distance requirement is dependent on the coil and the acceptable imitation error and might lay between 4 and 10R [21]. The magnetic flux density $\overrightarrow{\boldsymbol{b}}(\overrightarrow{\boldsymbol{r}})$ at a specified position $\overrightarrow{\boldsymbol{r}}$ depends on the vacuum permeability $\mu_{0}$, and the coil's magnetic dipole moment $\vec{m}$ with the number of windings $N$. Each vector $(\vec{r}, \vec{m})$ is comprised of the vector norm $(r, m)$ and the corresponding unit vector $\left(\overrightarrow{\boldsymbol{e}}_{\mathrm{r}}, \vec{e}_{\mathrm{m}}\right)$. This equation describes a time-invariant (DC) magnetic field, which yields an AC field, when modulated with the excitation signal $g(n)$ :

$$
\overrightarrow{\boldsymbol{b}}(\overrightarrow{\boldsymbol{r}})=\frac{\mu_{0}}{4 \pi r^{2}} \cdot \frac{3 \overrightarrow{\boldsymbol{r}}(\overrightarrow{\boldsymbol{m}} \circ \overrightarrow{\boldsymbol{r}})-\overrightarrow{\boldsymbol{m}} r^{2}}{r^{3}} \quad \text { with } \quad \overrightarrow{\boldsymbol{m}}=N_{\mathrm{w}} I_{\mathrm{rms}} R^{2} \pi \overrightarrow{\boldsymbol{e}}_{\mathrm{m}} .
$$

The magnetic flux density at the sensor's position is comprised of constant, distancedependent, and directivity-related contributions. This results in a nominal flux density $b_{0}$ with a field constant $a_{0}$, a $1 / r^{3}$ decay (path loss), and a corresponding loss due to directivity $\overrightarrow{\boldsymbol{\Theta}}\left(\vec{e}_{\mathrm{r}}\right)$.

$$
\overrightarrow{\boldsymbol{b}}(\overrightarrow{\boldsymbol{r}})=\underbrace{a_{0} I_{\mathrm{rms}}}_{b_{0}} \cdot \frac{1}{r^{3}} \cdot \overrightarrow{\boldsymbol{\Theta}}\left(\overrightarrow{\boldsymbol{e}}_{\mathbf{r}}\right)
$$

The nominal field $b_{0}$ determines the feasible magnetic flux density at a specified position. It is constrained by competing geometric and electric parameters, as only a limited number of windings $N_{\mathrm{W}}$ at a radius $R$ fit into a constrained space. A reduced wire 
cross section enables more windings, but increases the electrical impedance and vice versa. An increase in current is generally possible within the voltage range of the source, but is ultimately limited by thermal constraints.

\subsection{Sensor Characterization and Idealization}

This motion sensing approach uses exchange bias magnetoelectric delta-E cantilever sensors with a sensor element made of multiple layers of magnetostrictive FeCoSiB material and piezoelectric material on a silicon substrate [22]. A sensor element and a low-noise charge amplifier [23] together form the ME sensor system.

The ME cantilever sensor is a vector magnetometer [24]. Accordingly, it will pick up magnetic fields depending on the orientation of its sensitive axis $\vec{e}_{\mathrm{s}}$. Fabrication might cause a tilt between the physical long axis and the sensitive axis. Based on our observations while rotating the sensor in a Helmholtz coil, the tilt is very small (low single digit degrees maximum) for this specific sensor. Thus, the magnetic flux density $d(\vec{r})$ that the sensor picks up due to modulation might be modeled as the dot product of the sensor orientation and the magnetic vector field:

$$
d(\overrightarrow{\boldsymbol{r}})=\overrightarrow{\boldsymbol{e}}_{S} \circ \overrightarrow{\boldsymbol{b}}(\vec{r}) .
$$

The sensor characterization measurements were done in the optimized conditions of a magnetically shielded measurement setup [25]. Therefore, real-world measurements will show an inferior sensor performance. The selected frequency space is targeted at the first bending mode close to $7.7 \mathrm{kHz}$, which is specified by the mechanical properties of the cantilever. Delta-E effect readout schemes for much lower frequencies [9] are also possible for this type of sensor, but not used in this application.

Based on the assumption of Linearity and time invariance (LTI), the measured frequency response $\hat{H}\left(e^{j \Omega}\right)$ (Figure 3a) quantifies the conversion of a magnetic field into a voltage. It is synonymous with the sensor's sensitivity in this context.

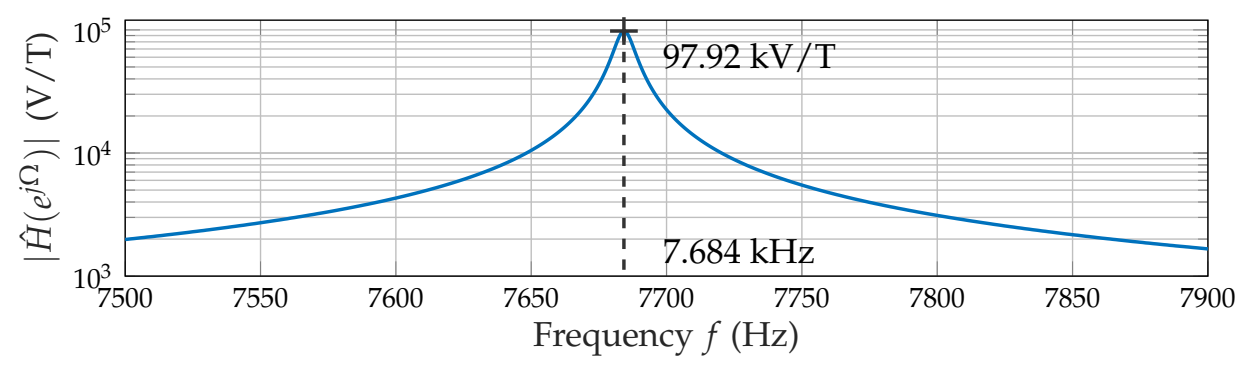

(a) Sensitivity curve with peak sensitivity at the resonance frequency.

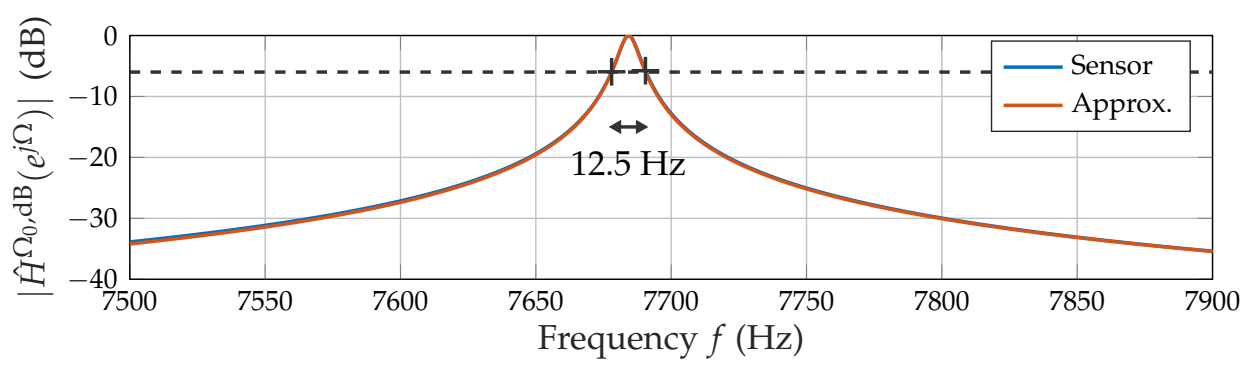

(b) Normalized filter characteristic with IIR peak filter defined by $6 \mathrm{~dB}$ bandwidth.

Figure 3. Frequency response of the ME sensor.

The sensitivity curve (Figure 3a) results from multiple discrete measurement points interpolated using a spline method. The data were obtained using a magnetic sine sweep at a peak amplitude of $100 \mathrm{nT}$. The peak sensitivity (approximately $100 \mathrm{kV} / \mathrm{T}$ ) is reached at the resonance frequency (approximately $7.684 \mathrm{kHz}$ ). The filter characteristic (Figure 3b) of 
the sensor is obtained from the logarithmic amplitude response normalized to the peak sensitivity $\hat{H}^{\Omega_{0}, \mathrm{~dB}}\left(e^{j \Omega}\right)$. The terminology for discrete-time signals with the normalized angular frequency $\Omega$ and the corresponding resonance frequency $\Omega_{0}$ leads to:

$$
\left|\hat{H}^{\Omega_{0}, \mathrm{~dB}}\left(e^{j \Omega}\right)\right|=20 \log _{10}\left(\frac{\left|\hat{H}\left(e^{j \Omega}\right)\right|}{\left|\hat{H}\left(e^{j \Omega_{0}}\right)\right|}\right) .
$$

The resulting $6 \mathrm{~dB}$ bandwidth of $12.5 \mathrm{~Hz}$ and the resonance frequency were adopted to design a digital filter that approximates the filter characteristic in the region of interest. Based on a priori knowledge of the mechanical cantilever, an infinite impulse response (IIR) peak (resonator) filter [26] was chosen (Figure 3b).

The voltage noise amplitude spectral density (ASD) $\hat{A}_{\mathrm{vv}}(\Omega)$ (Figure $4 \mathrm{a}$ ) is the other crucial performance metric obtained from the ME sensor characterization. It enables the estimation of the noise power at specific frequencies.

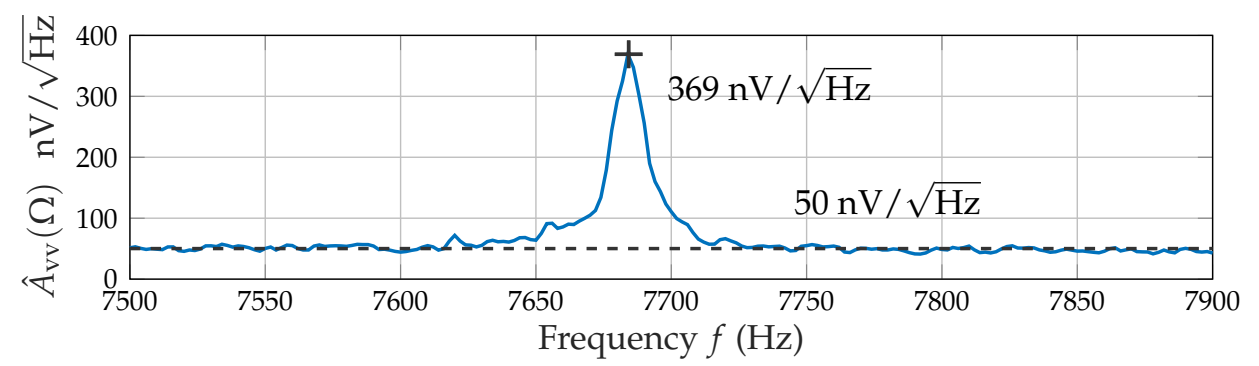

(a)

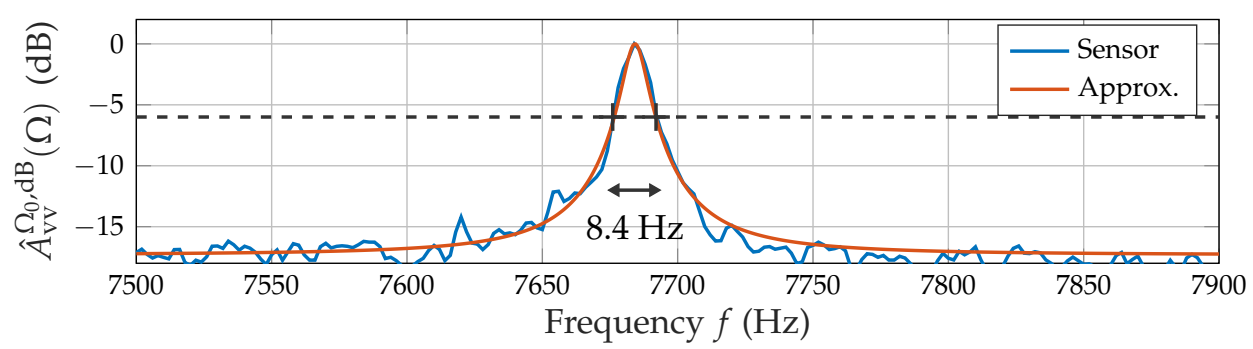

(b)

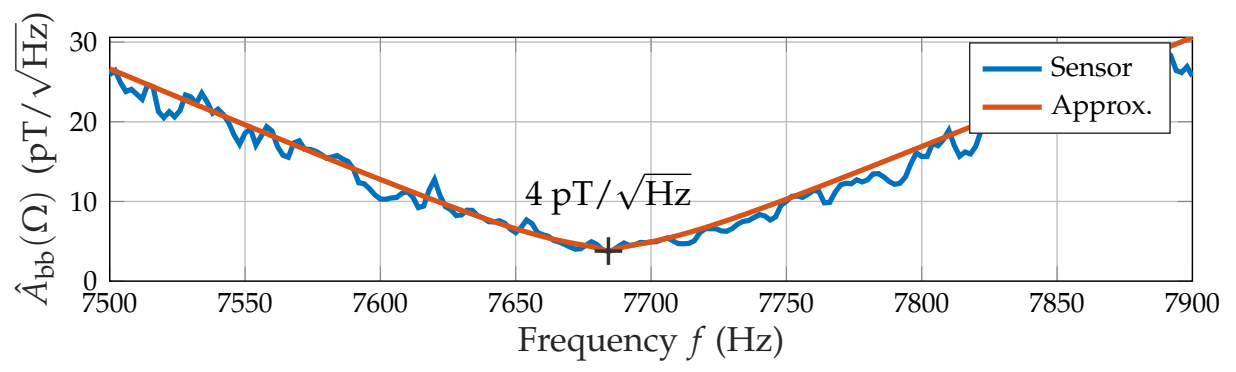

(c)

Figure 4. ME sensor noise characteristics. (a) Voltage noise spectral density with peak and floor value. (b) Normalized noise filter with IIR peak filter approximation defined by $6 \mathrm{~dB}$ bandwidth. (c) Magnetic noise density with minimum and IIR peak filter approximation.

$\hat{A}_{\mathrm{vv}}(\Omega)$ (Figure $4 \mathrm{a}$ ) reaches its local maximum of $370 \mathrm{nV} / \sqrt{\mathrm{Hz}}$ at the sensor's resonance frequency and approaches a floor level of approximately $50 \mathrm{nV} / \sqrt{\mathrm{Hz}}$. The filter characteristic is again obtained from the logarithmic noise density by normalizing it to the peak noise value in resonance: 


$$
\hat{A}_{\mathrm{VV}}^{\Omega_{0}, \mathrm{~dB}}(\Omega)=20 \log _{10}\left(\frac{\hat{A}_{\mathrm{vV}}(\Omega)}{\hat{A}_{\mathrm{vV}}\left(\Omega_{0}\right)}\right) .
$$

The filter characteristic is approximated similarly to the frequency response by using an IIR peak (boost) filter [27] that employs a parallel allpass structure to limit the stopband's attenuation. The required design parameters include the boost factor (ratio between passband and stopband) and the $6 \mathrm{~dB}$ bandwidth of $8.4 \mathrm{~Hz}$ (Figure $4 \mathrm{~b}$ ). The sensor's performance in measuring magnetic fields (magnetic noise density $\hat{A}_{\mathrm{bb}}(\Omega)$ ) is dependent on both frequency response and voltage noise density:

$$
\hat{A}_{\mathrm{bb}}(\Omega)=\frac{\hat{A}_{\mathrm{vv}}(\Omega)}{\left|\hat{H}\left(e^{j \Omega}\right)\right|} .
$$

The resulting spectrum (c) presents a minimum in noise density of $4 \mathrm{pT}$ at resonance with approximately linear slopes to both sides. Some form of magnetic noise density value (at a varying application-dependent frequency, e.g., [10]) is commonly used as an important performance metric for magnetometers. The curve is approximated by multiplying the inverse frequency response approximation and the voltage noise density approximation.

\subsection{Signal Processing Structure}

As previously characterized, the sensor offers a very limited $6 \mathrm{~dB}$ bandwidth of $12.5 \mathrm{~Hz}$. Generally, it is beneficial for the SNR to adapt the bandwidth depending on the target application's requirements. Thus, an equalizing filter corresponding to the inverse approximated frequency response was applied, which resulted in a flat frequency response in the region of interest. $\hat{A}_{\mathrm{bb}}(\Omega)$ (Figure $4 \mathrm{c}$ ) is suitable for noise performance estimations, where a deviation from the sensor's $6 \mathrm{~dB}$ bandwidth is required. This is practically realized by some form of band limiting.

The sensor also picked up a multitude of undesired magnetic and electric fields as it was operated unshielded in the experimental setup. This effect was even worsened by the forceful amplification of low frequencies due to the equalizer. It might be countered by a band limiting to the operating range of $f_{0} \pm 200 \mathrm{~Hz}$, which was subsequently applied using a 10th order Butterworth filter.

The final noise reduction step is a matched filter which corresponds to a bandwidth limitation and demodulation based on knowledge of the excitation signal $g(n)$. The length of the matched filter $T_{\mathrm{mf}}$ ( $N$ samples) is an assumption on how long the desired signal $d(n)$ is approximately constant. In the stationary case, one might choose a very long (e.g., multiple seconds) and therefore narrow matched filter to achieve a superior noise performance. In the 1D motion case $(>0.1 \mathrm{~m} / \mathrm{s})$, the assumption of a constant $d(n)$ for such a filter is invalid. Consequently, the matched filter distorts the estimation result $\hat{d}(k)$ by suppressing high frequency components of $d(n)$.

In general, the trade-off between a high spatial accuracy for slow movements (such as the localization of a resting object) and a high temporal accuracy for fast movements (such as obtaining the high-frequency components of movement) has to be managed. A matched filter implementation with the (equivalent) correlator realization [28] might be beneficial here, as it enables the parallel use of a multitude of filters with different lengths $N_{j}$. Thereby, the process of multiplying (weighting) the enhanced signal $p(n)$ with the excitation signal $g(n)$ can be separated from the summation and is only required once. The multiplication requires a matching phase (group delay for non-sinusoidal signals). Processing is done at a lower common sample (update) rate $f_{\text {up }}$ (period: $T_{\text {up }}$ ) of $100 \mathrm{~Hz}$ which is linked to the system sample rate $f_{s}$ of $48 \mathrm{kHz}$ by the conversion factor $c$. Figure $5 \mathrm{a}$ illustrates this process in principle:

$$
\hat{d}_{j}(k)=\frac{1}{N_{j}} \sum_{i=0}^{N_{j}-1} p(k-i) g(k-i) \quad \text { with } \quad k=c n .
$$


Our specific implementation for measurements (Figure 5a) covers two competing matched filters:

(1) $T_{\mathrm{mf}, 1}$ targets "fast" movements with a filter length of $10 \mathrm{~ms}$, which corresponds to a two-sided bandwidth of $100 \mathrm{~Hz}$.

(2) $T_{\mathrm{mf}, 2}$ targets "slow" movements with a filter length of $200 \mathrm{~ms}$, which corresponds to a two-sided bandwidth of $5 \mathrm{~Hz}$.

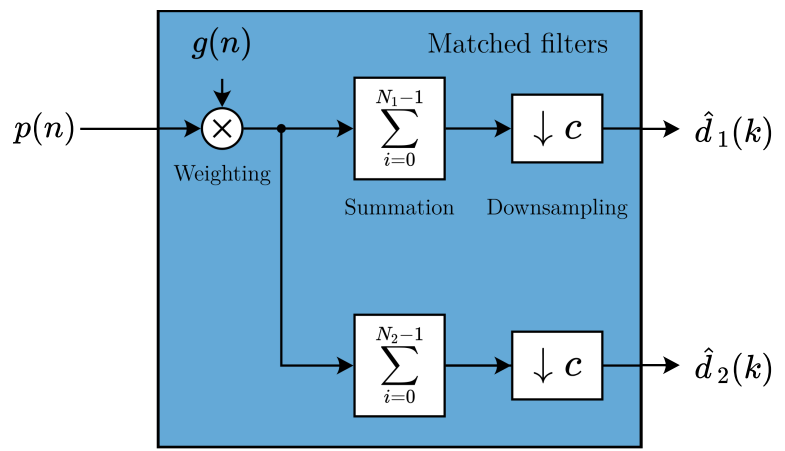

(a) Single-channel matched filter structure.

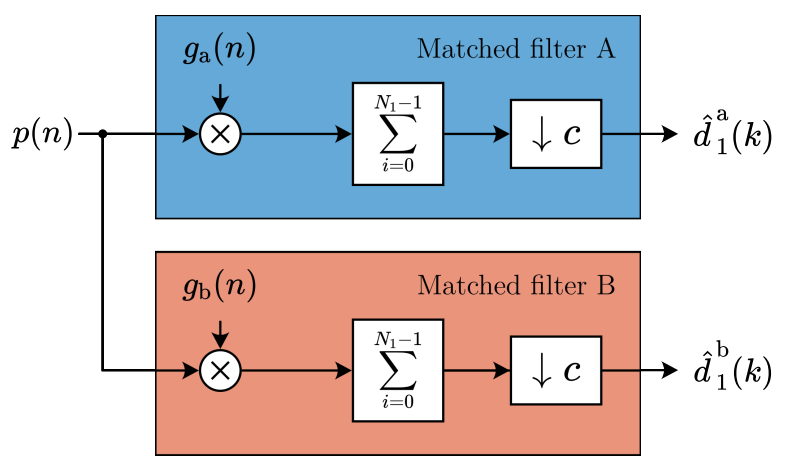

(c) Matched filters for FDMA.

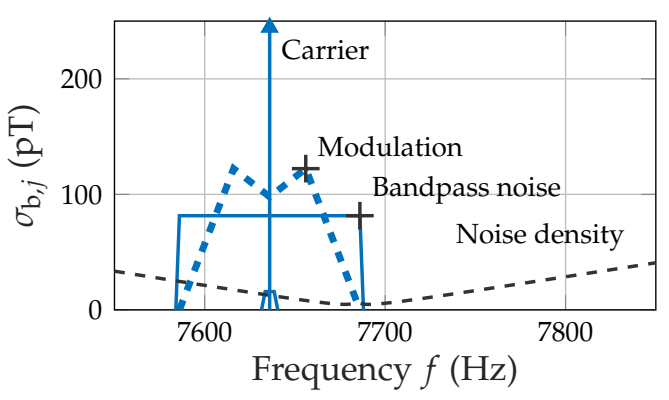

(b) Bandpass noise for single channel.

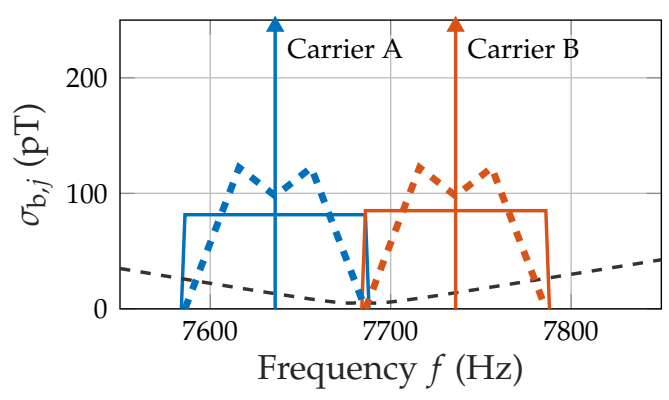

(d) Bandpass noise for FDMA.

Figure 5. Matched filter structures and corresponding bandwidth considerations.

Integration of the magnetic noise density (ideal conditions, cf. Figure 4c) within the specified matched filter bandwidth illustrates the effect of the matched filter length (Figure 5b). The carrier is placed at $7636 \mathrm{~Hz}$ (approximately $f_{0}-50 \mathrm{~Hz}$ ). Modulation due to motion will require additional bandwidth apart from the carrier. Integration within an ideal bandpass is illustrated here as a rectangle with a width (integration bandwidth) and a height (square root of the integrated noise power $\sigma_{\mathrm{b}, j}^{2}$ ). $T_{\mathrm{mf}, 1}$ yields an approximate theoretical noise of $80 \mathrm{pT}$, while $T_{\mathrm{mf}, 2}$ yields a significantly lower noise below $16 \mathrm{pT}$.

This matched filter implementation also allows for the separation of multiple simultaneously active carriers in a frequency division multiple access (FDMA) scheme (Figure 5c). The weighting signals $g_{\mathrm{a}, \mathrm{b}}(n)$ correspond to both active carriers. Subsequently, various matched filter lengths (e.g., $N_{1}$ ) might be applied in parallel. Figure $5 \mathrm{~d}$ illustrates the placement of both carriers $100 \mathrm{~Hz}$ apart. 


\subsection{Simulation}

In addition to the physical setup, we set up a simulation to provide a reference signal $\tilde{d}(k)$ for the estimation result $\hat{d}(k)$. As the processing strictly separates the excitation signal $g(n)$ from the desired magnetic signal $d(n)$, the simulation only includes a DC magnetic field based on a dipole approximation and an idealized 1D motion in this field:

$$
\tilde{d}(k)=\hat{\overrightarrow{\boldsymbol{e}}}_{s} \circ \underbrace{\hat{a}_{0} \hat{I}_{\mathrm{rms}}}_{\hat{b}_{0}} \cdot \frac{1}{\hat{r}^{3}} \cdot \overrightarrow{\boldsymbol{\Theta}}\left(\hat{\overrightarrow{\boldsymbol{e}}}_{r}\right) \text { with } \quad \hat{\vec{r}}(k) .
$$

Figure 6 visualizes how the previously characterized parameters of the overall system are included in the simulation model. In comparison to the physical setup, there is no bandpass filter applied, so even fast simulated movements will not lead to a signal distortion.

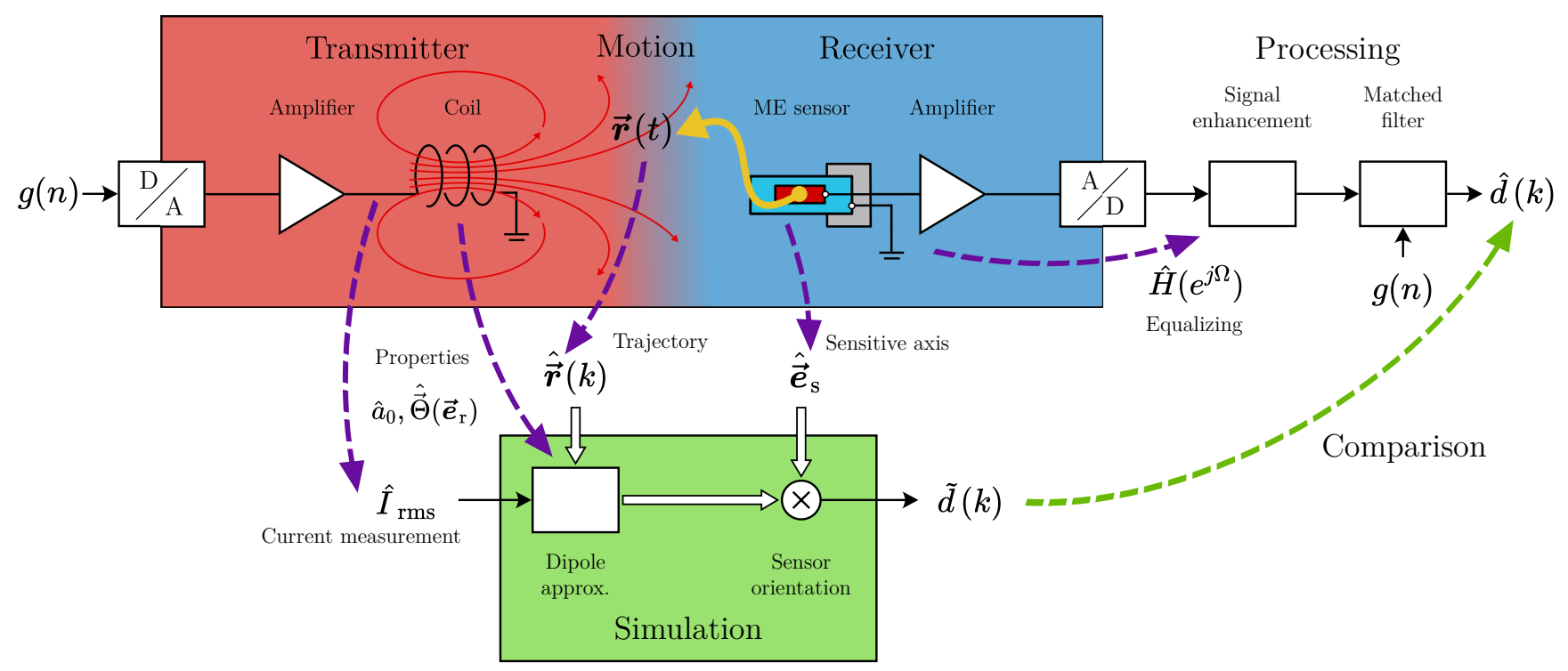

Figure 6. Overall signal chain including simulation.

\section{Measurements and Results}

\subsection{Measurement Setup}

Measurements were conducted in a simplified motion scenario (Figure 7) on a $1.2 \mathrm{~m}$ aluminum track. The sensor system is moving on a cart towed by stepper motors with its long axis $\hat{\overrightarrow{\boldsymbol{e}}}_{s}$ in the direction of movement. The velocity $\dot{x}$ in the central part of the track $(97 \mathrm{~cm})$ is assumed to be constant. With a starting point $x_{0}$ and a $y_{0}$ displacement of $10 \mathrm{~cm}$ to each coil, the subsequent equation for the relative position is adopted for the field simulation:

$$
\hat{\vec{r}}(k)=\left(\begin{array}{c}
x(k) \\
y_{0} \\
0
\end{array}\right)=\left(\begin{array}{c}
\dot{x} \cdot k T_{\mathrm{up}}+x_{0} \\
y_{0} \\
0
\end{array}\right) .
$$

The setup features two cylindrical coils for magnetic excitation fields powered by two composite amplifiers (based on [29]) at a current $\hat{I}_{\text {rms }}$ of approximately $400 \mathrm{~mA}$. Optical switches close to the beginning and the end of the track enable synchronization and average velocity measurements. The conversion of signals between the analog and the digital domain was done using a sound card (UFX+, RME). The basic validity of the simulated magnetic flux density along the track was verified using a fluxgate sensor (FLC 100, Stefan Mayer Instruments). 


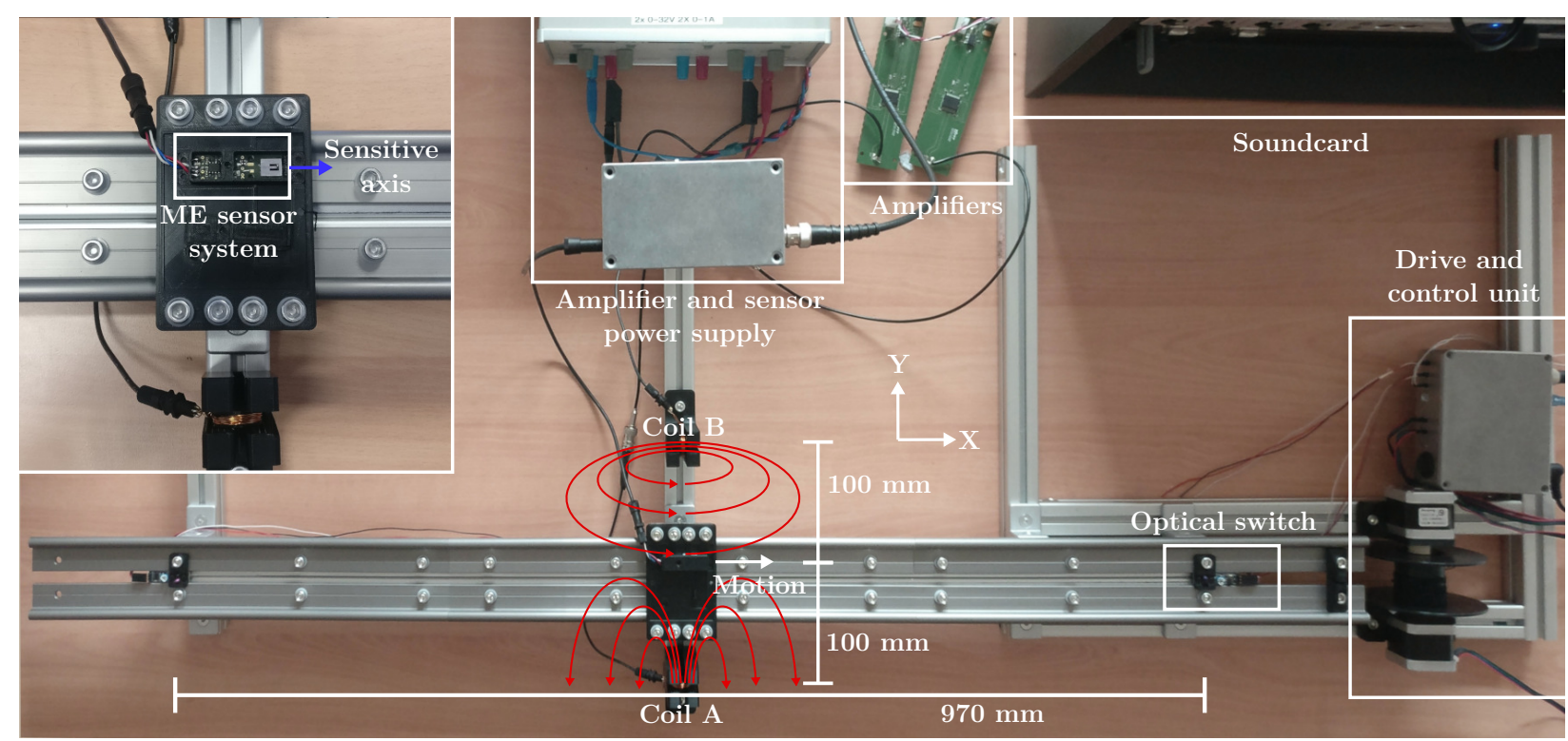

(a)

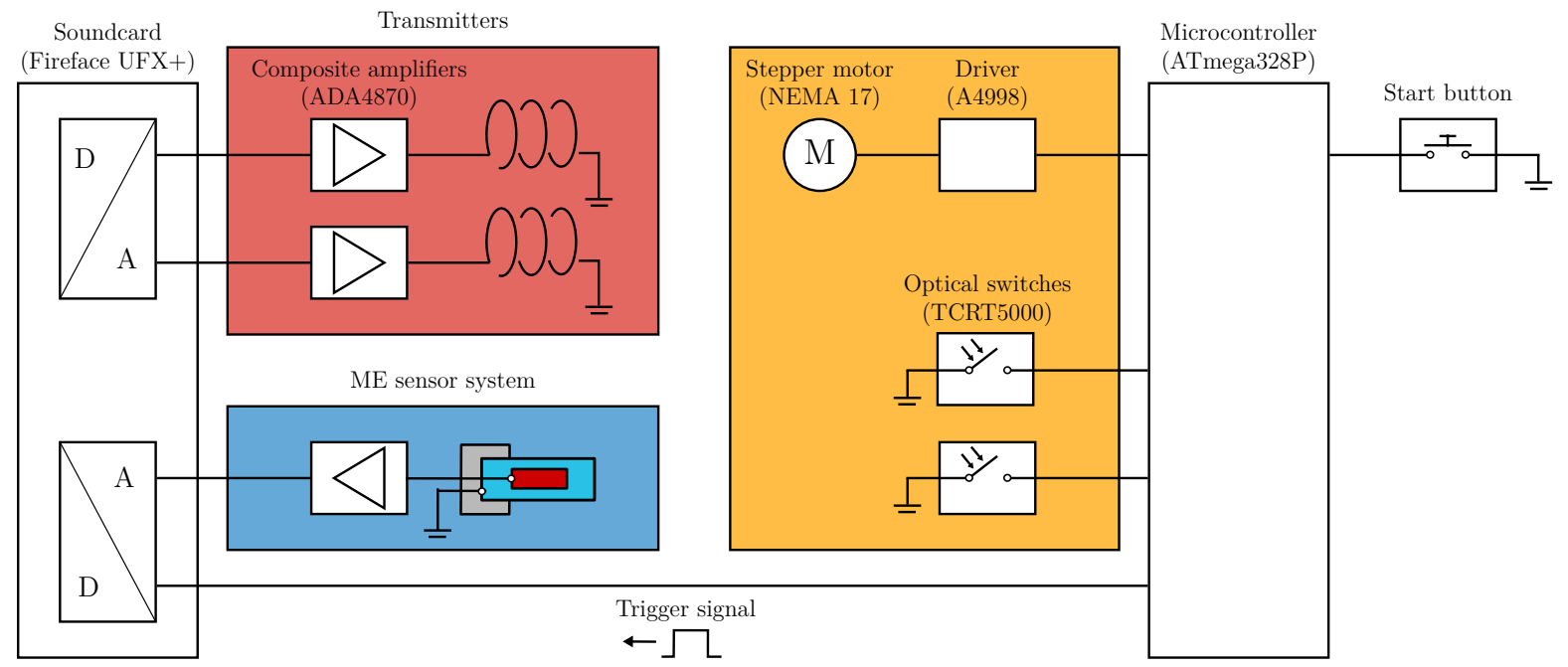

(b)

Figure 7. Experimental setup for magnetoelectric motion sensing. (a) Overview of the experimental setup. (b) Block diagram of key functional components.

\subsection{Single Channel Measurements}

As a first proof of concept, only coil A was used to generate a magnetic field, while the sensor was moving along the track multiple times at varying velocities. Based on the optical switches' trigger signals, each desired signal's time scale was rescaled to distance. As only the $x$ position of the sensor is time-variant, we also refer to the measurement/simulation results as $\hat{d}(x)$ and $\tilde{d}(x)$, respectively. The previously introduced matched filter lengths $N_{1}$ and $N_{2}$ were applied on each signal to manage the trade-off between bandwidth and noise. Figure 8a shows accordance between the simulated signal and all three measured signals. However, the signals appear to be quite noisy. In Figure 8b, there is less noise but the signal measured at the fastest average velocity of $0.62 \mathrm{~m} / \mathrm{s}$ is significantly flattened. 


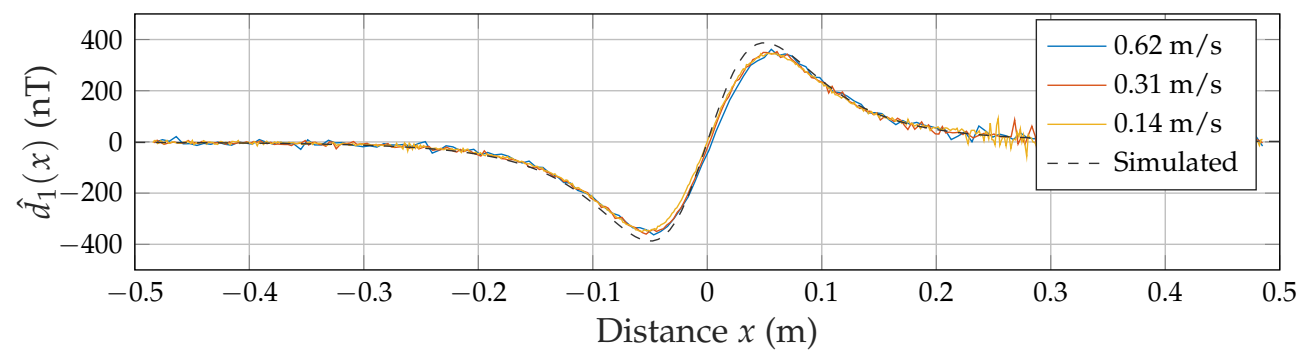

(a) Matched filter length of $10 \mathrm{~ms}$.

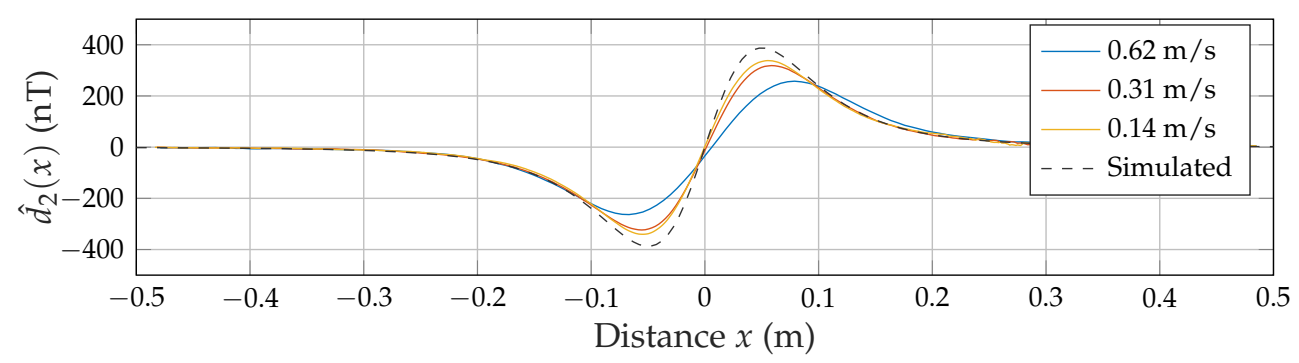

(b) Matched filter length of $200 \mathrm{~ms}$.

Figure 8. Measured signals for varying speeds and matched filter lengths.

\subsection{Noise Measurements}

As we established the receiver noise as the main limitation of system performance in the previous chapter, we conducted noise measurements (without magnetic excitation) of resting and moving sensors on the test setup (cf. Figure 8). Three different scenarios were tested and processed using the previously introduced matched filter of length $T_{\mathrm{mf}, 1}$ and $T_{\mathrm{mf}, 2}$, respectively. The resting case (Figure $9 \mathrm{a}, \mathrm{b}$ ) is thereby compared with a slower movement (Figure 9c) at the higher MF length and with a faster movement (Figure 9d) at the shorter MF length. For each measurement, the corresponding noise standard deviation (e.g., $\left.\sigma_{\mathrm{u}, 1}^{\mathrm{a}}\right)$ is provided.

While the resting case $\left(\sigma_{\mathrm{u}, 1}^{\mathrm{a}}\right.$ and $\left.\sigma_{\mathrm{u}, 2}^{\mathrm{b}}\right)$ shows a very low noise close to the spectral integration (cf. Figure $5 \mathrm{~b}$ ), the moving cases yield a significant increase in noise (approximately 100 times) due to spikes $\left(\sigma_{\mathfrak{u}, 1}^{\mathrm{c}}\right.$ and $\left.\sigma_{\mathrm{u}, 2}^{\mathrm{d}}\right)$. The assumption of a stationary noise power $\left(\sigma_{\mathrm{u}, 1}^{\mathrm{c}}\right)$ during the motion is only valid for some sections of the time signal, as oscillation and multiple prominent peaks occur.

Based on the obtained noise values $\sigma_{\mathrm{u}, 1}^{\mathrm{a}}$ to $\sigma_{\mathrm{u}, 2}^{\mathrm{d}}$, multiple performance metrics regarding range and accuracy were calculated. Firstly, the noise floor was directly plotted in comparison to the positive half-wave of the simulated magnetic field (Figure 10a, cf. Figure 8). This leads directly to the SNR calculation as depicted in Figure 10b.

$$
\operatorname{SNR}_{1}^{\mathrm{a}}(\vec{r})^{\mathrm{dB}}=20 \log _{10}\left(\frac{\tilde{d}(\vec{r})}{\sigma_{\mathrm{u}, 1}^{\mathrm{a}}}\right)
$$

While $\sigma_{\mathrm{u}, 1}^{\mathrm{a}}$ and $\sigma_{\mathrm{u}, 2}^{\mathrm{b}}$ show significant SNR levels even at at distance of $50 \mathrm{~cm}$, the SNR reaches zero quickly for both other scenarios at $33 \mathrm{~cm}\left(\sigma_{\mathfrak{u}, 1}^{\mathrm{c}}\right)$ and $25 \mathrm{~cm}\left(\sigma_{\mathrm{u}, 2}^{\mathrm{d}}\right)$, respectively. The noise can also be added on top of the simulated magnetic curve to obtain the uncertainty in the measurement of magnetic flux density for each position. This results in an upper and a lower boundary between which the measured value is to expected at the specified confidence of one standard deviation (Figure 10c). It is now possible to reverse search the corresponding spatial values $\hat{x}$ for the upper and the lower boundary at each position. This results in an upper and a lower estimated distance value. Error bars are included for relevant values of 1 and $5 \mathrm{~cm}$ (Figure 10d). While $\sigma_{\mathrm{u}, 2}^{\mathrm{b}}$ ensures a high accuracy of $1 \mathrm{~cm}$ even 
at a distance of $42 \mathrm{~cm}, \sigma_{\mathfrak{u}, 1}^{\mathrm{c}}$ reaches $1 \mathrm{~cm}$ at a distance of $15 \mathrm{~cm}$ and $\sigma_{\mathfrak{u}, 2}^{\mathrm{d}}$ never even reaches $1 \mathrm{~cm}$.

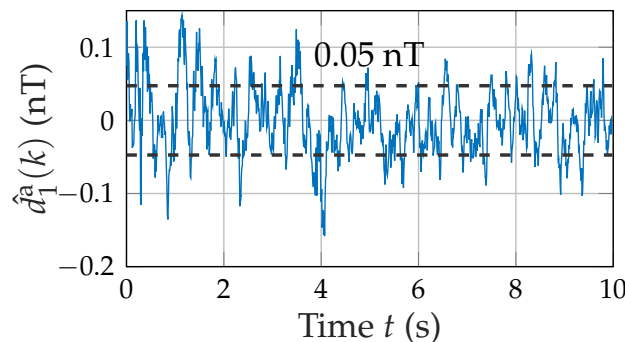

(a) Sensor resting $\left(T_{\mathrm{mf}, 1}\right)$.

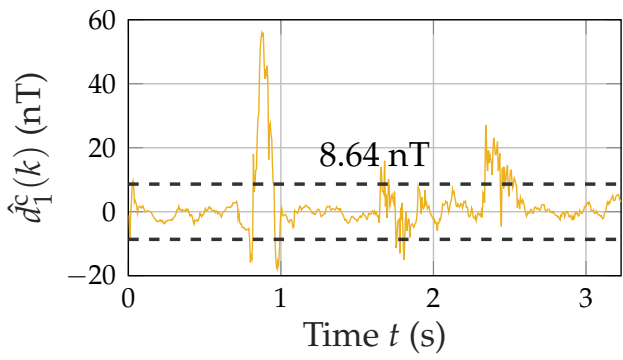

(c) Sensor moving at $0.31 \mathrm{~m} / \mathrm{s}\left(T_{\mathrm{mf}, 1}\right)$.

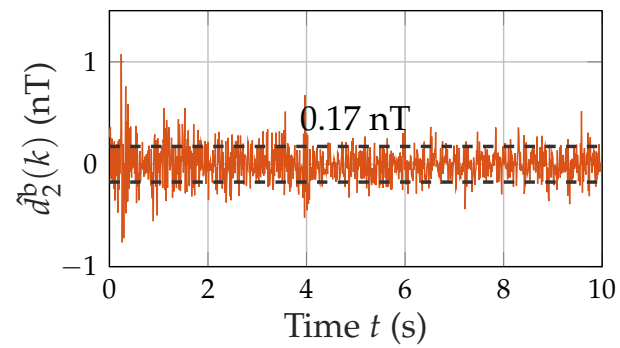

(b) Sensor resting $\left(T_{\mathrm{mf}, 2}\right)$.

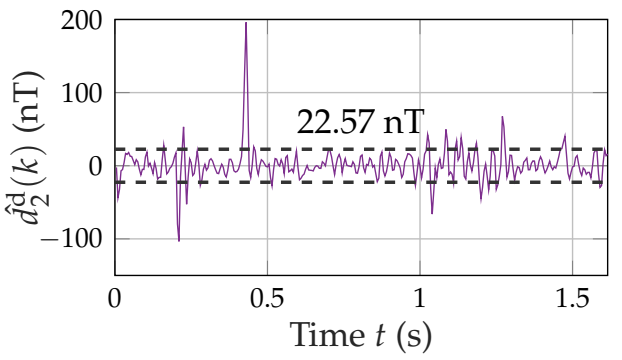

(d) Sensor moving at $0.62 \mathrm{~m} / \mathrm{s}\left(T_{\mathrm{mf}, 2}\right)$.

Figure 9. Noise measurements of moving and resting sensors. Each plot contains the noise at the matched filter output and the corresponding RMS value from $\sigma_{\mathrm{u}, 1}^{\mathrm{a}}$ to $\sigma_{\mathrm{u}, 2}^{\mathrm{d}}$.

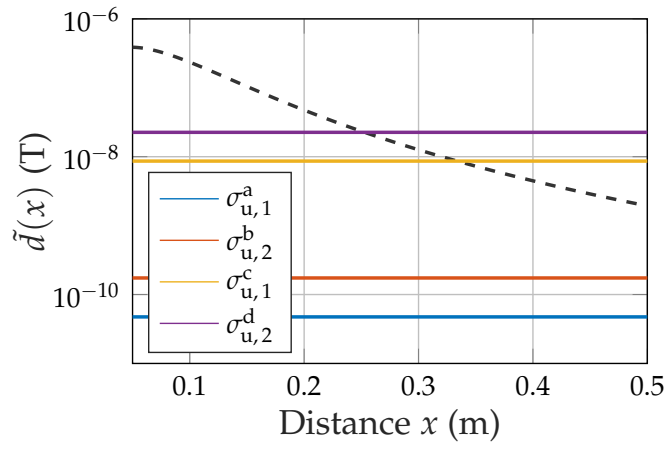

(a) Range considerations.

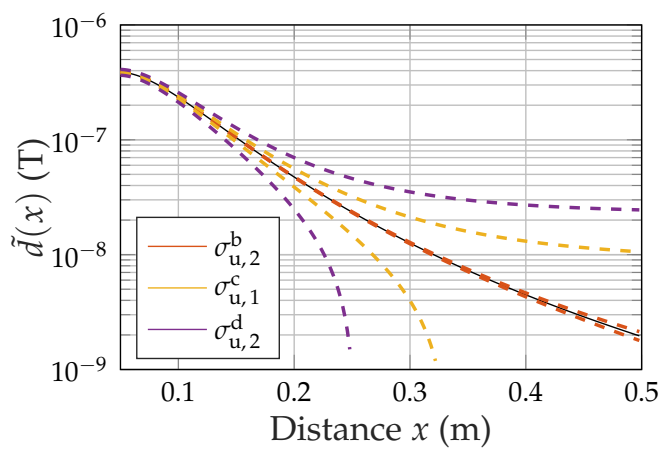

(c) Magnetic error.

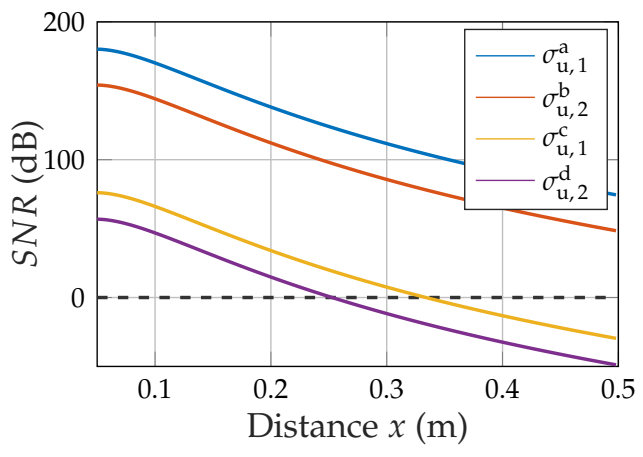

(b) Signal-to-noise ratio.

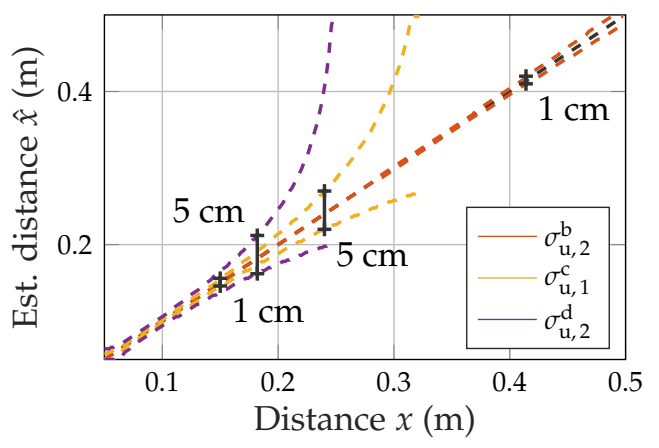

(d) Spatial error.

Figure 10. Performance metrics based on noise. 


\subsection{Frequency Division Multiple Access Measurement}

The FDMA approach utilizes the second excitation coil to transmit another carrier signal in a separate frequency band. As coil B is rotated by $90^{\circ}$ compared to coil A, the sensor measures the axial component of this magnetic field. Figure 11 shows the capability of the applied matched filter structure to smoothly separate both signals at the applied velocity of $0.31 \mathrm{~m} / \mathrm{s}$ (with a matched filter length $T_{\mathrm{mf}, 1} \mathrm{of} 5 \mathrm{~ms}$ ) even with the high dynamic difference between both signals at a distance $x$ close to zero.

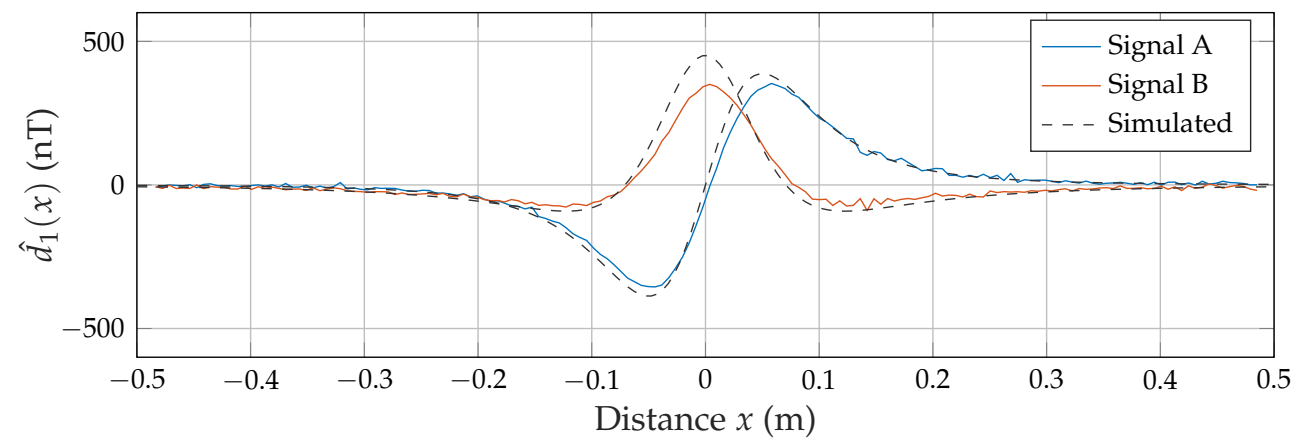

Figure 11. Estimated desired signals in an FDMA approach.

\section{Conclusions and Outlook}

In this article, we presented a concept for magnetic motion sensing using ME sensors and multiple artificial sources. We focused on signal-to-noise ratio as the key metric to determine the performance of this approach and showed the basic feasibility in an experimental setup.

The setup was targeted at the demonstration of sensor measurements during a defined movement. While the restriction of motion to a single axis is suitable for characterization, it does not cover the full range of a $6 \mathrm{D}$ tracking problem, where position and orientation of the sensor might change. The solution of such a problem might require supplemental sensors and sources.

The first proof-of-concept measurements using a single or dual excitation signal generally show accordance with the magnetic simulation. A visual comparison between both scenarios shows no significant influence of intercarrier interference. While the magnetic simulation for coil A matched quite well, there was a more significant deviation for coil B. We generally expected some uncertainty for both coils regarding the exact measurement of the current and the estimation of position, orientation, and geometric properties of the coil (e.g., mean radius). In particular, the current might vary between each coil as well as between physical setup and simulation. This is due to the low impedance (cf. Figure 2), which is consequently affected by parasitic resistances of connectors and cables as well as measurement inaccuracy.

In contrast to the localization in the quasi-stationary case, we considered bandwidth as a key factor in motion sensing. The proposed structure uses multiple matched filters in parallel, which define the bandwidth requirements. The selection of the instantaneous bandwidth manages the trade-off between a high spatial accuracy for slow movements and a sufficient temporal accuracy for fast movements. There might be potential in an adaptive selection based on velocity or position estimations.

The noise measurements during motion showed a significant increase in noise compared to the resting case, which also leads to a degradation in SNR. Some of the noiseincreasing effects might be related to the experimental nature of the measurement setup. As the sensor cable is trailing the cart, it might cause mechanical oscillations, which propagate to the sensor. This is relevant for the peaks that regularly occurred during the measurements. It also affected the theoretical range of $25 \mathrm{~cm}$ for the highest bandwidth, which was below our initial expectations. These performance metrics (cf. Figure 10) were obtained for an approximately constant movement. When considering the bandwidth only, they 
should be comparable for any arbitrary motion with similar bandwidth characteristics in its corresponding magnetic (desired) signal. However, there might be other mechanical effects in practical measurements that contribute to the additional noise.

As receiver noise was identified to be the key factor for system performance, there are multiple potential improvements conceivable: a cantilever without a magnetostrictive layer (piezoelectric sensor [30]) might be employed to analyze the effects of mechanical noise due to motion and apply adaptive noise reduction schemes; electrical shielding might improve noise performance at the cost of sensitivity and a tilted sensitivity axis (as in [9]); additionally, an increased matched filter length is an option for slow and quasi-stationary movements.

However, we assessed magnetoelectric motion sensing as a preliminary development stage of a comprehensive human motion tracking system. This does not yet allow for a meaningful comparison with common motion tracking systems. Instead, the SNR is more of a ground floor, which can potentially be significantly improved by subsequent tracking and sensor fusion algorithms (e.g., extended Kalman-based) if a priori knowledge on the motion and the moving object is taken into account.

All of the applied scenarios were focused on simple movements that are not generally comparable to the complexity of human movements. As we aim for an application in movement analysis for neurodegenerative diseases like Parkinson's disease, an important next step is the application of a magnetoelectric motion sensing setup inside the clinical motor lab with our partners at the University Medical Center Schleswig-Holstein. State-ofthe-art optical and IMU reference systems are available there to validate the magnetoelectric measurement results and evaluate the system's performance. As the signal processing is currently done offline, such measurements would also greatly benefit from a real-time implementation.

Author Contributions: Conceptualization, J.H. and E.E.; methodology, J.H.; software, J.H.; validation, J.H.; formal analysis, J.H.; investigation, J.H. and C.B.; data curation, J.H.; writing—original draft preparation, J.H. ; writing—review and editing, J.H., E.E., C.B. and G.S.; visualization, J.H.; supervision, E.E., C.B. and G.S.; project administration, W.M. and G.S.; funding acquisition, C.H., W.M. and G.S. All authors have read and agreed to the published version of the manuscript.

Funding: This work was supported by the German Research Foundation (Deutsche Forschungsgemeinschaft, DFG) through the project B9 of the Collaborative Research Centre CRC 1261 "Magnetoelectric Sensors: From Composite Materials to Biomagnetic Diagnostics".

Institutional Review Board Statement: Not applicable.

Informed Consent Statement: Not applicable.

Data Availability Statement: Not applicable.

Acknowledgments: The authors would like to thank Henrik Wolframm for providing the composite amplifiers and Christine Kirchhof and Phillip Durdaut for creating the sensor.

Conflicts of Interest: The authors declare no conflict of interest. The funders had no role in the design of the study; in the collection, analyses, or interpretation of data; in the writing of the manuscript, or in the decision to publish the results.

\section{Abbreviations}

The following abbreviations are used in this manuscript:

ME Magnetoelectric

IMU Inertial measurement unit

SNR Signal-to-noise ratio

OPM Optically pumped magnetometer

SQUID Superconducting quantum interference device

DC Direct current 


$\begin{array}{ll}\text { AC } & \text { Alternating current } \\ \text { VLF } & \text { Very low frequency } \\ \text { TDMA } & \text { Time division multiple access } \\ \text { CDMA } & \text { Code division multiple access } \\ \text { FDMA } & \text { Frequency division multiple access } \\ \text { RMS } & \text { Root mean square } \\ \text { ASD } & \text { Amplitude spectral density } \\ \text { MF } & \text { Matched filter }\end{array}$

\section{References}

1. Sabatini, A.M. Kalman-Filter-Based Orientation Determination Using Inertial/Magnetic Sensors: Observability Analysis and Performance Evaluation. Sensors 2011, 11, 9182-9206. [CrossRef]

2. Kortier, H.G.; Sluiter, V.I.; Roetenberg, D.; Veltink, P.H. Assessment of hand kinematics using inertial and magnetic sensors. J. Neuroeng. Rehabil. 2014, 11, 70. [CrossRef]

3. Mirelman, A.; Bernad-Elazari, H.; Thaler, A.; Giladi-Yacobi, E.; Gurevich, T.; Gana-Weisz, M.; Saunders-Pullman, R.; Raymond, D.; Doan, N.; Bressman, S.B.; et al. Arm swing as a potential new prodromal marker of Parkinson's disease. Mov. Disord. 2016, 31, 1527-1534. [CrossRef]

4. $\quad$ Espay, A.J.; Bonato, P.; Nahab, F.; Maetzler, W.; Dean, J.M.; Klucken, J.; Eskofier, B.M.; Merola, A.; Horak, F.; Lang, A.E.; et al. Technology in Parkinson disease: Challenges and Opportunities. Mov. Disord. 2016, 31, 1272-82. [CrossRef]

5. Thun-Hohenstein, C.; Klucken, J. Wearables as a Supportive Tool in the Care of Patients with Parkinson's Disease: A Paradigm Change. Klin. Neurophysiol. 2021, 52, 44-51. [CrossRef]

6. Roetenberg, D.; Slycke, P.J.; Veltink, P.H. Ambulatory Position and Orientation Tracking Fusing Magnetic and Inertial Sensing. IEEE Trans. Biomed. Eng. 2007, 54, 883-890. [CrossRef] [PubMed]

7. Pasku, V.; Angelis, A.D.; Angelis, G.D.; Arumugam, D.D.; Dionigi, M.; Carbone, P.; Moschitta, A.; Ricketts, D.S. Magnetic field-based positioning systems. IEEE Commun. Surv. Tutor. 2017, 19, 2003-2017. [CrossRef]

8. Bosch Sensortec GmbH. BMX055 IMU Specification. Available online: https://www.bosch-sensortec.com/products/motionsensors/absolute-orientation-sensors/bmx055/ (accessed on 3 August 2021).

9. Spetzler, B.; Bald, C.; Durdaut, P.; Reermann, J.; Kirchhof, C.; Teplyuk, A.; Meyners, D.; Quandt, E.; Höft, M.; Schmidt, G.; et al. Exchange biased delta-E effect enables the detection of low frequency pT magnetic fields with simultaneous localization. Sci. Rep. 2021, 11, 5269. [CrossRef] [PubMed]

10. Stefan Mayer Instruments. FL1-100 Fluxgate Magnetometer Specification. Available online: https://stefan-mayer.com/de/ produkte/magnetometer-und-sensoren/magnetfeldsensor-fl1-100.html (accessed on 22 November 2021).

11. QuSpin. QTFM Total Field Magnetometer Specification. Available online: https://quspin.com/qtfm/ (accessed on 3 August 2021).

12. Twinleaf. microSAM Total Field Magnetometer Specification. Available online: https://twinleaf.com/scalar/microSAM/ (accessed on 3 August 2021).

13. Wu, F.; Liang, Y.; Fu, Y.; Ji, X. A robust indoor positioning system based on encoded magnetic field and low-cost IMU. In Proceedings of the IEEE/ION Position, Location and Navigation Symposium (PLANS), Savannah, GA, USA, 11-14 April 2016; pp. 204-212. [CrossRef]

14. Hellmers, H.; Norrdine, A.; Blankenbach, J.; Eichhorn, A. An IMU/magnetometer-based Indoor positioning system using Kalman filtering. In Proceedings of the International Conference on Indoor Positioning and Indoor Navigation, Montbeliard, France, 28-31 October 2013. [CrossRef]

15. Polhemus. Viper Motion Tracking System. Available online: https://polhemus.com/viper (accessed on 3 August 2021).

16. Caruso, M.J.; Bratland, T.; Smith, C.H.; Schneider, R. A new perspective on magnetic field sensing. Sensors 1998, 15, 34-47.

17. Tumanski, S. Handbook of Magnetic Measurements; CRC Press Taylor and Francis: Boca Raton, FL, USA, 2011 ; pp. 6, 42, 45.

18. Omicron Labs. Vector Network Analyzer Bode 100 Rev. 1. 2021. Available online: https://www.omicron-lab.com/products/ vector-network-analysis/bode-100/technical-data (accessed on 3 August 2021).

19. Bao, J.; Hu, C.; Lin, W.; Wang, W. On the magnetic field of a current coil and its localization. In Proceedings of the 2012 IEEE International Conference on Automation and Logistics, Zhengzhou, China, 15-17 August 2012; pp. 573-577. [CrossRef]

20. Rothwell, E.J.; Cloud, M.J. Electromagnetics; CRC Press: Boca Raton, FL, USA, 2001; pp. 158-160.

21. Paperno, E.; Plotkin, A. Cylindrical induction coil to accurately imitate the ideal magnetic dipole. Sens. Actuators A Phys. 2004, 112, 248-252. [CrossRef]

22. Gojdka, B.; Jahns, R.; Meurisch, K.; Greve, H.; Adelung, R.; Quandt, E.; Knöchel, R.; Faupel, F. Fully integrable magnetic field sensor based on delta-E effect. Appl. Phys. Lett. 2011, 99, 223502. [CrossRef]

23. Durdaut, P.; Penner, V.; Kirchhof, C.; Quandt, E.; Knöchel, R.; Höft, M. Noise of a JFET Charge Amplifier for Piezoelectric Sensors. IEEE Sens. J. 2017, 17, 7364-7371. [CrossRef]

24. Lage, E.; Woltering, F.; Quandt, E.; Meyners, D. Exchange biased magnetoelectric composites for vector field magnetometers. J. Appl. Phys. 2013, 113, 17C725. [CrossRef] 
25. Jahns, R.; Knöchel, R.; Greve, H.; Woltermann, E.; Lage, E.; Quandt, E. Magnetoelectric sensors for biomagnetic measurements. In Proceedings of the 2011 IEEE International Symposium on Medical Measurements and Applications, Bari, Italy, 30-31 May 2011, pp. 107-110. [CrossRef]

26. Proakis, J.G.; Manolakis, D.G. Digital Signal Processing: Principles, Algorithms and Applications; Prentice Hall: Upper Saddle River, NJ, USA, 1996; pp. 340-343.

27. Zölzer, U. Digital Audio Signal Processing; Wiley: Hoboken, NJ, USA, 2008; p. 129.

28. Sklar, B. Digital Communications: Fundamentals and Applications; Number 5; Prentice Hall: Upper Saddle River, NJ, USA, 2007; pp. 122-126.

29. Analog Devices. ADA4870, High Speed, High Voltage, 1 A Output Drive Amplifier. 2021. Available online: https://www.analog. $\mathrm{com} / \mathrm{media} / \mathrm{en} /$ technical-documentation/data-sheets/ADA4870.pdf (accessed on 3 August 2021).

30. Reermann, J.; Bald, C.; Salzer, S.; Durdaut, P.; Piorra, A.; Meyners, D.; Quandt, E.; Höft, M.; Schmidt, G. Comparison of reference sensors for noise cancellation of magnetoelectric sensors. In Proceedings of the 2016 IEEE SENSORS, Orlando, FL, USA, 30 October-3 November 2016; pp. 1-3. [CrossRef] 